\title{
Nuclear kinematics in nearby AGN - I. An ALMA perspective on the morphology and kinematics of the molecular $\mathrm{CO}(2-1)$ emission
}

\author{
V. Ramakrishnan ${ }^{\oplus},{ }^{1 \star}$ N. M. Nagar, ${ }^{1}$ C. Finlez, ${ }^{1}$ T. Storchi-Bergmann, ${ }^{2}$ R. Slater,,${ }^{1,3}$ \\ A. Schnorr-Müller, ${ }^{2}$ R. A. Riffel ${ }^{\oplus},{ }^{4}$ C. G. Mundell ${ }^{5}$ and A. Robinson ${ }^{6}$ \\ ${ }^{1}$ Astronomy Department, Universidad de Concepción, Casilla 160-C, 4030000 Concepción, Chile \\ ${ }^{2}$ Departamento de Astronomia, Instituto de Física, Universidade Federal do Rio Grande do Sul, $91501-970$ Porto Alegre, RS, Brazil \\ ${ }^{3}$ Dirección de Formación General, Facultad de Educación y Cs. Sociales, Universidad Andres Bello, Sede Concepción, autopista Concepción-Talcahuano \\ 7100, Talcahuano, Chile \\ ${ }^{4}$ Departamento de Física, Centro de Ciências Naturais e Exatas, Universidade Federal de Santa Maria, $97105-900$ Santa Maria, RS, Brazil \\ ${ }^{5}$ Department of Physics, University of Bath, Claverton Down, Bath BA2 7AY, UK \\ ${ }^{6}$ School of Physics and Astronomy, Rochester Institute of Technology, University of Rochester, 85 Lomb Memorial Drive, Rochester, NY 14623, USA
}

Accepted 2019 May 2. Received 2019 April 30; in original form 2018 June 4

\begin{abstract}
We present the molecular gas morphology and kinematics of seven nearby Seyfert galaxies obtained from our $230 \mathrm{GHz}$ ALMA observations. The $\mathrm{CO} \mathrm{J}=2-1$ kinematics within the inner $\sim 30 \operatorname{arcsec}(\lesssim 9 \mathrm{kpc})$ reveals rotation patterns that have been explored using the Bertola rotation model and a modified version of the Kinemetry package. The latter algorithm reveals various deviations from pure circular rotation in the inner kiloparsec of all seven galaxies, including kinematic twists, decoupled, and counter-rotating cores. A comparison of the global molecular gas and stellar kinematics shows overall agreement in the position angle of the major axis and the systemic velocity, but larger discrepancies in the disc inclination. The residual maps obtained with both the methods show the presence of non-circular motions in most of the galaxies. Despite its importance, a detailed interpretation of the physics responsible for non-circular motions will be discussed in a forthcoming work.
\end{abstract}

Key words: galaxies: active-galaxies: kinematics and dynamics-galaxies: nucleigalaxies: Seyfert.

\section{INTRODUCTION}

The mechanism that drives the energy output from an active galaxy is understood to be governed by the process of accretion on to a supermassive black hole (SMBH). Several works have shown a correlation between the SMBH mass and the overall properties of the spheroidal component of the host galaxy (Ferrarese \& Merritt 2000; Gebhardt et al. 2000; Tremaine et al. 2002). One such significant finding is the connection between the black hole mass and the velocity dispersion of the host spheroid (Gültekin et al. 2009), which supports the connection between the build-up of stellar mass of a galaxy and its SMBH. A fundamental problem here is in understanding the processes that govern the evolution of the black hole and its effect on the host galaxy.

Feeding the SMBH requires gas to be driven from galaxy scales down to sub-kpc and finally sub-pc scales: in galaxy discs this effectively requires the gas to lose angular momentum. Mechanisms for driving gas to the nucleus could be secular, e.g. due to the

^E-mail: vramakrishnan@udec.cl presence of non-axisymmetric nuclear structures such as the spiral arms with different extent of warping, oval distortions and bars, (Kormendy \& Kennicutt 2004; Maciejewski 2004; Hopkins \& Quataert 2010), or due to external influences, e.g. galaxy interactions and major or minor mergers (Sanders et al. 1988; Quinn, Hernquist \& Fullagar 1993; Kauffmann \& Haehnelt 2000; Di Matteo, Springel \& Hernquist 2005; Hopkins et al. 2008). For a review on observations of inflows on different spatial scales see Storchi-Bergmann \& Schnorr-Müller (2019).

An important process by which the SMBH effects the evolution of the galaxy is 'feedback' - the deposition of mechanical and/or radiative energy from the SMBH into the interstellar medium. The magnitude of this feedback is regulated in part by the magnitude of the accretion flows on to the black hole. The spatial scale over which this process occurs spans several orders of magnitude in distance. The current understanding of this process in a galaxy from a cosmological perspective remains an enigma. It is, therefore, important to study the effects of the feedback on a galaxy at both small and large scales.

There is prolific evidence for molecular gas outflows from luminous active galaxies. This outflowing matter is cold interstellar 
gas that affects the evolution of the host galaxy by fuelling the star formation and accretion on to the central black hole (Morganti, Tadhunter \& Oosterloo 2005; Alatalo et al. 2011; Cicone et al. 2014; Tadhunter et al. 2014; Morganti et al. 2016)

Over the past decades, deep observations of galaxies have provided us with an insight into galaxy evolution and structure formation in the Universe. Following these results, the enhanced star formation and nuclear activity in galaxies have been revealed in greater detail by high-resolution broad-band imaging and longslit spectroscopic surveys. With technological advancements, we are now in the era of integral-field spectroscopy, which provides a high resolution both in the spectral and spatial domain. It is thus possible to efficiently map the ionized gas, stars, star formation rates, and other parameters with a high precision using integral-field units (IFU). In order to improve our understanding of the physical processes and dynamical structures in galaxies, it is critically necessary to combine the stellar and ionized gas kinematics obtained with IFUs with those of molecular gas; the imaging spectroscopy of millimetre $(\mathrm{mm})$ interferometers, such as the Atacama Large Millimeter Array (ALMA) provides the latter.

Several previous works have studied the kinematics of ionized and/or molecular gas in nearby galaxy samples. The best example for the former is the SAURON (de Zeeuw et al. 2002) and CALIFA surveys (Sánchez et al. 2012) that exploited the IFU on the William Herschel and Calar Alto telescopes, respectively. The kinematics of the molecular (typically $\mathrm{CO}$ ) emission was studied in detail by surveys like NUGA (García-Burillo et al. 2003), ATLAS ${ }^{3 D}$ (Cappellari et al. 2011; Young et al. 2011; Alatalo et al. 2013), and EDGE-CALIFA (Bolatto et al. 2017; Levy et al. 2018) using IRAM and CARMA observations. A majority of the galaxies show a disc-like morphology in which rotation dominates, though non-axisymmetric kinematics are often seen, and sometimes even dominate. Although these previous surveys cover a large number of galaxies, the quality of their kinematic results was hampered by the limited sensitivity and mapping capability of the interferometers used. The study of feedback in galaxies got a significant boost with the commissioning of the ALMA interferometer. The superior CO maps from ALMA in Seyfert galaxies like NGC 1566 revealed noncircular motions from a bar, indicating strong negative torques on gas at $r=100-300$ pc (Combes et al. 2014; Slater et al. 2019). High velocity outflows have also been observed in other galaxies like NGC 1433 (Combes et al. 2013), NGC 1377 (Aalto et al. 2012), and IC 5063 (Morganti et al. 2015).

This paper is organized as follows. We discuss the overall objectives of our project and the sample selection in Section 2, and the observations and the data processing in Section 3. The models utilized in this work are discussed in detail in Section 4 followed by the results in Section 5 . The discussion and the summary of this work are given in Section 6 and detailed descriptions of the results for each sample galaxy are listed in Section 7.

\section{OBJECTIVES AND SAMPLE SELECTION}

The growing evidence for a correlation between nuclear dust structures and accretion activity supports the hypothesis that nuclear spirals are a mechanism for fuelling the SMBH (e.g. Emsellem et al. 2001; Maciejewski et al. 2002; Crenshaw, Kraemer \& Gabel 2003; Fathi et al. 2005; Simões Lopes et al. 2007). Our group has analysed optical and infrared IFU data of several active galaxies, selected for having dusty nuclear structures. These observations reveal streaming motions of ionized and molecular gas towards the nucleus along dusty spiral arms and/or ionized gas outflows (e.g. Riffel et al. 2008; Riffel, Storchi-Bergmann \& Winge 2013; Schnorr-Müller et al. 2014a; Diniz et al. 2015; Schnorr-Müller et al. 2017; Humire et al. 2018).

We thus undertook a pilot study of nine active galaxies that were selected for: (i) showing signatures of strong streaming inflows, outflows, and/or bright (IR) molecular gas discs in our previous IFU observations; (ii) being close enough to resolve structures at scales of tens of parsecs; and (iii) (typically) having previous detections of nuclear $\mathrm{CO}$ emission at a lower resolution. These galaxies were observed using ALMA to trace the CO J $=2-1$ emission.

Thus, in conjunction with our IFU maps of the stellar and ionized gas morphology and kinematics, and high-resolution NIR imaging, we attempt to quantify the high-resolution $\mathrm{CO}(2-1)$ observations of seven nuclei from our sample in this work. The remaining two, viz. NGC 1566 (Slater et al. 2019) and NGC 3393 (Finlez et al. 2018), have already been analysed in detail. The sample galaxies, and their basic parameters, are listed in Table 1.

\section{OBSERVATIONS AND DATA REDUCTION}

All galaxies were observed with ALMA during Cycles 2 (projectID 2012.1.00474.S; PI - Nagar) and 4 (project-ID 2015.1.00086.S; PI - Nagar). The observations were taken using the ALMA Band 6 receivers on 32 12-meter antennas. Four spectral windows (SPWs), two in the lower sideband and two in the upper sideband were used. Three of these were configured to cover the following lines at relatively high resolution $\left(\sim 2.6 \mathrm{~km} \mathrm{~s}^{-1}\right)$ : ${ }^{12} \mathrm{CO}(2-1)$ $\left(v_{\text {obs }}=229.401922 \mathrm{GHz}\right),{ }^{13} \mathrm{CH} 3 \mathrm{OH}\left(v_{\mathrm{obs}}=241.548041 \mathrm{GHz}\right)$, and CS J $=5-4\left(v_{\mathrm{obs}}=243.728532 \mathrm{GHz}\right)$. The remaining SPW was set to lower spectral resolution in order to better detect continuum emission. The SPWs were thus centred on 229.415, 227.060, 241.554, and $243.735 \mathrm{GHz}$, with bandwidths of 1.875, 2.0, 1.875, and $1.875 \mathrm{GHz}$, respectively, and spectral resolutions of 2.6, 20.53, 2.6 , and $2.6 \mathrm{~km} \mathrm{~s}^{-1}$, respectively.

The flux and the phase calibrators used for every source along with the total observing time and the synthesized beam size are listed in Table 2. Data were calibrated and imaged using CASA 4.7.0 (McMullin et al. 2007). For every source, the continuum emission in the uv-plane was subtracted from the SPWs using the task uvcontsub. The cell sizes used for every source while imaging are given in Table 2 . The $\mathrm{CO}(2-1)$ emission line was strongly detected in all sources over a velocity range of $\sim \pm 200 \mathrm{~km} \mathrm{~s}^{-1}$, and we were able to map the CO line at a channel spacing of $2.6 \mathrm{~km} \mathrm{~s}^{-1}$.

Strong nuclear continuum emission was detected in all sources except NGC 1667. Therefore, it was necessary to subtract the continuum emission from the $u v$ data before imaging the molecular line emission. To improve the fidelity and dynamic range of the final data cube, both amplitude and phase self-calibration solutions that were obtained during the process of making the continuum maps was applied to the spectral line data before imaging. The $\mathrm{CO}(2-1)$ spectral line was then imaged using a natural weighting at a spectral channel spacing of $2.6 \mathrm{~km} \mathrm{~s}^{-1}$ for all sources.

\subsection{Moment maps}

The data cubes obtained above are a series of images stacked along a spectral dimension. In order to facilitate further interpretation, it is useful to create $2 \mathrm{D}$ maps through a linear combination of individual planes. In this work, we generate three such maps (as is a common practice in the field of feedback studies) with each dependent on the sum (integrated intensity, $M_{0}$ ), average (integrated velocity, $M_{1}$ ), and the standard deviation (velocity dispersion, $M_{2}$ ) 
Table 1. List of sample galaxies. Morphology and the nucleus type are obtained from Malkan, Gorjian \& Tam (1998). Source position and the redshift are from SIMBAD (which are in turn obtained from the Two Micron All Sky Survey, hereafter 2MASS; Skrutskie et al. 2006). The luminosity distance and scale were estimated assuming a flat cosmology with $\mathrm{H}_{0}=67.8, \Omega_{M}=0.3$, and $\Omega_{\Lambda}=0.7$ (Planck Collaboration XIII 2016). The position angle (PA) and the inclination (i) are from the 2MASS catalogue.

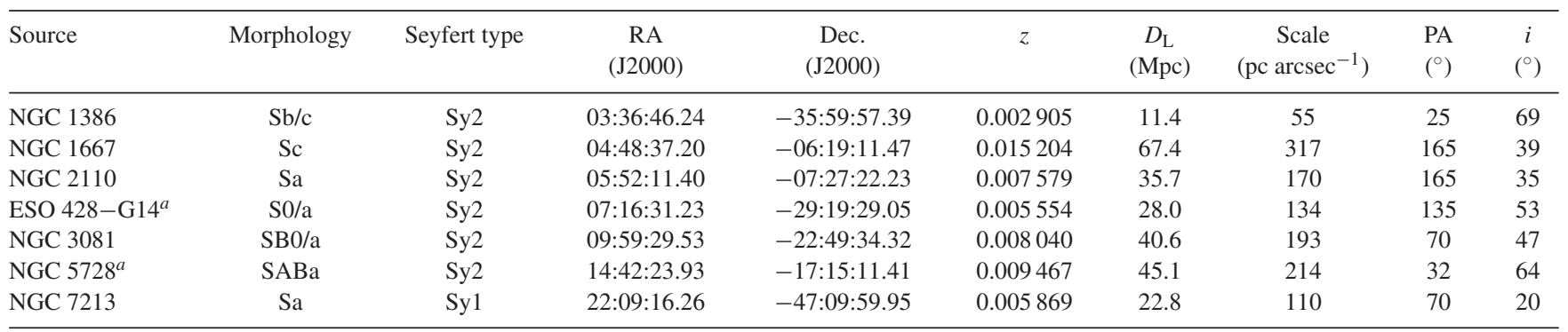

${ }^{a}$ Morphology and the nucleus type are obtained from de Vaucouleurs et al. (1991).

Table 2. Observing parameters.

\begin{tabular}{|c|c|c|c|c|c|c|c|}
\hline Source & Date of observation & Flux calibrator & Bandpass/Phase calibrator & $\begin{array}{l}\text { Total observing time } \\
\qquad(\min )\end{array}$ & $\begin{array}{l}\text { Cell size } \\
(\operatorname{arcsec})\end{array}$ & $\begin{array}{c}\mathrm{FWHM}^{a} \\
(\operatorname{arcsec} \times \operatorname{arcsec}) \\
\left({ }^{\circ}\right)\end{array}$ & $\begin{array}{c}\mathrm{rms}^{b} \\
\left(\mathrm{mJy} \mathrm{km} \mathrm{s}^{-1}\right)\end{array}$ \\
\hline NGC 1667 & 2014 Jul 08 & J0423-013 & J0423-0120 & 45.4 & 0.09 & $0.55 \times 0.51(66)$ & 0.7 \\
\hline NGC 2110 & 2015 May 14 & Ganymede & J0423-0120/J0541-0541 & 45.0 & 0.06 & $0.99 \times 0.56(-69)$ & 0.9 \\
\hline ESO 428-G14 & 2016 May 12 & $\mathrm{~J} 0750+1231$ & J0538-4405/J0648-3044 & 35.7 & 0.06 & $0.80 \times 0.66(-57)$ & 0.8 \\
\hline NGC 3081 & 2016 May 02 & J1107-4449 & J1037-2934/J0927-2034 & 36.2 & 0.06 & $0.82 \times 0.59(-62)$ & 1.5 \\
\hline NGC 7213 & 2014 May 27 & Neptune & J2056-4714/J2235-4835 & 44.0 & 0.06 & $0.62 \times 0.57(81)$ & 0.8 \\
\hline
\end{tabular}

${ }^{a}$ Major and minor axes of the synthesized beam, and its PA (North to East).

${ }^{b}$ Noise per $2.6 \mathrm{~km} \mathrm{~s}^{-1}$ channel.

along the frequency dimension. The respective maps and their errors were generated using equation (1). The maps are computed with a threshold that defines the pixel values to be included. For all the sources in this work, we generated moment maps assuming a threshold of $4 \sigma$ with the noise level shown in Table 2. All channels with visible $\mathrm{CO}$ emission (typically 250 to 600 channels) were used in the calculation of the moment maps. We note that the galaxies discussed in this work primarily show rotation dominated, rather than dispersion dominated, kinematics. Thus the standard deviation $\left(M_{2}\right)$ maps trace both the intrinsic dispersion of the gas, and the rotation velocity gradient across the synthesized beam. For simplicity, we refer to the $M_{2}$ maps as the 'velocity dispersion' maps, even though they show the upper limit of the intrinsic velocity dispersion especially in the nuclear regions where the rotation velocity gradients are relatively large.

In equation (1), the intensity at a given pixel for a channel velocity is given by $I(v)$ and the channel separation (or velocity width) by $\Delta v$. The term $w$ corresponds to $\sum I(v)$.

The moment maps of NGC 1386 are shown in Fig. 1 while those of other sources are shown in Appendix B, which is provided online. For all sources except NGC 1386, the integrated CO map peak(s) are not cospatial with the $230 \mathrm{GHz}$ continuum peak (assumed to be the nuclear position). Sources NGC 1667 (Fig. B1; online) and NGC 5728 (Fig. B5; online) contain two such CO-bright regions within the inner $\sim 2$ arcsec which symmetrically straddle the nucleus. Spiral arms are evident in the integrated $\mathrm{CO}$ emission maps of all sources except for ESO 428-G14 and NGC 5728. These arms, shown as green contours in the Hubble Space Telescope (HST) images, seem to overlap quite well with the spiral dust lanes seen in the HST images. The moment one maps of all sources show a clear velocity gradient with an indication of a rotating component. This motion coupled with the disc-like rotation that can immediately be inferred from the maps of ESO 428-G14 and NGC 5728 gives rise to the classic double-horn shape of the integrated spectrum (Figs B3 and B6; online).

$$
\begin{aligned}
M_{0}= & \Delta v \sum I(v) \\
M_{0, \mathrm{err}}= & \sqrt{\sum I_{\mathrm{err}}^{2}(v)} \\
M_{1}= & \frac{\sum v I(v)}{w} \\
M_{1, \mathrm{err}}= & \sqrt{\sum\left(\frac{w v-\sum v I(v)}{w^{2}}\right)^{2} \times I_{\mathrm{err}}^{2}(v)} \\
M_{2}= & \sqrt{\frac{\sum\left(v-M_{1}\right)^{2} I(v)}{w}} \\
M_{2, \mathrm{err}}= & \left\{\left(\frac{w\left(v-M_{1}\right)^{2}-\sum\left(I(v)\left(v-M_{1}\right)^{2}\right)}{w^{2}}\right)^{2} \times I_{\mathrm{err}}^{2}(v)\right. \\
& \left.+\left(\frac{2 \sum\left(I(v)\left(v-M_{1}\right)\right)}{w}\right)^{2} \times M_{1, \mathrm{err}}^{2}\right\}^{1 / 2}
\end{aligned}
$$

\section{2 $230 \mathrm{GHz}$ continuum maps}

The peak flux of the $230 \mathrm{GHz}$ continuum emission of the sample galaxies is in the range $\sim 1-5 \mathrm{mJy}$. NGC 1386 , NGC 5728 , and 

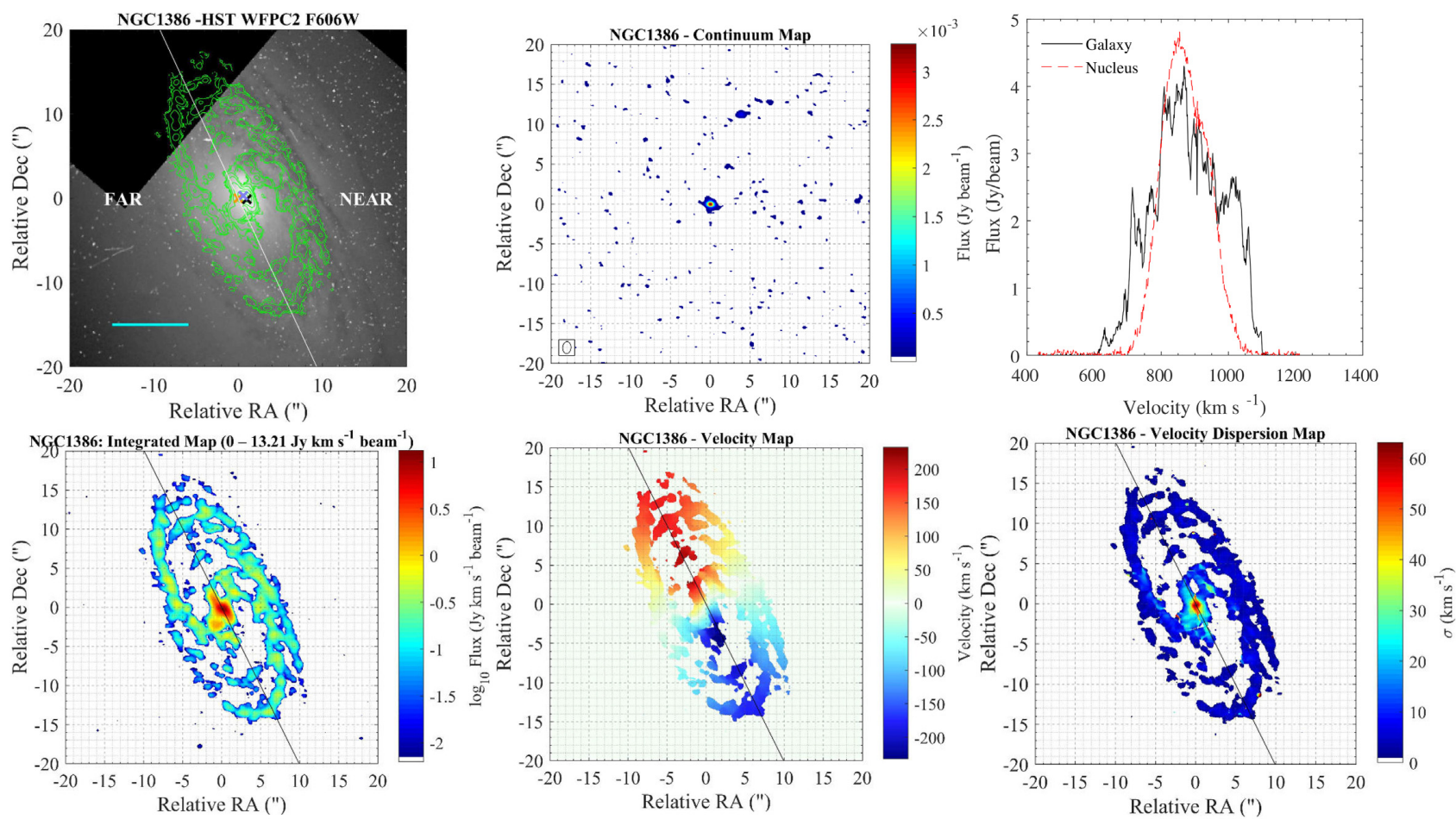

Figure 1. NGC 1386. Top-row, from left to right: An HST image (filter provided in the title), ALMA 230 GHz continuum map, and the galaxy-integrated $\mathrm{CO}(2-1)$ spectrum (black) along with the nuclear spectrum (red) extracted from an aperture of radius equal to half the size of the major axis of the synthesized beam. Bottom-row: Maps of the integrated flux in logarithmic scale $\left(\mathrm{Jy} \mathrm{km} \mathrm{s}^{-1}\right)$, velocity $\left(\mathrm{km} \mathrm{s}^{-1}\right)$, and velocity dispersion $\left(\mathrm{km} \mathrm{s}{ }^{-1}\right)$ of the $\mathrm{CO}(2-1)$ line. The crosses in the HST map corresponds to the centres from the $230 \mathrm{GHz}$ continuum (purple), $\mathrm{CO}(2-1)$ kinematics (red), HST optical peak (black), and SIMBAD-listed position (orange), while the green contours overlayed are the respective $\mathrm{CO}(2-1)$ integrated map. The white line in the HST map is the photometric major axis while the black one in the moment maps (bottom-row) corresponds to the kinematic major axis. The blue horizontal solid line in the bottom-left corner of the HST image is a scale bar that corresponds to $500 \mathrm{pc}$.

NGC 7213 show an unresolved $230 \mathrm{GHz}$ core, while ESO 428-G14, NGC 2110 (Fig. B2; online), and NGC 3081 (Fig. B4; online) show extended structure. The elongation of this continuum structure is seen almost along the major axis of the disc in the case of ESO 428-G14 (Falcke et al. 1996) and NGC 2110 (Mundell et al. 2000); in the latter case we are clearly detecting the well-known $\mathrm{S}$-shaped jet previously seen at lower frequencies. In NGC 3081 the continuum is extended along the minor axis culminating in a secondary shock feature (Nagar et al. 1999). The two shocked regions thus visible in the continuum map of NGC 3081 could correspond to the two brightest features seen in the nuclear region of the HST map. The only source with no detection of $230 \mathrm{GHz}$ continuum emission is NGC 1667.

\section{KINEMATIC DECOMPOSITION METHODS}

\subsection{Bertola model}

We first model the velocity maps of all galaxies with a purerotation model. Specifically, we assume circular orbits in a spherical potential as given in Bertola et al. (1991).

$$
\begin{aligned}
& V_{\text {mod }}(R, \psi)=V_{\text {sys }} \\
& +\frac{A R \cos \left(\psi-\psi_{0}\right) \sin (\theta) \cos ^{p}(\theta)}{\left\{R^{2}\left[\sin ^{2}\left(\psi-\psi_{0}\right)+\cos ^{2} \theta \cos ^{2}\left(\psi-\psi_{0}\right)\right]+c_{0}^{2} \cos ^{2} \theta\right\}^{p / 2}}
\end{aligned}
$$

Here, $V_{\text {sys }}$ is the systemic velocity, $A$ the amplitude of the rotation curve, $R$ and $\psi$ are the radial and angular coordinates for a given pixel in the plane of the sky, $\psi_{0}$ is the position angle of the line of nodes, and $\theta$ is the inclination of the disc. The parameter $p$ denotes the mass distribution of the galaxy such that for a $p=1$ the rotation curve is asymptotically flat while enclosing the total mass of the system for a $p=1.5$. The concentration parameter $c_{0}$ corresponds to the radius at which the velocity reaches 70 per cent of the maximum amplitude.

In order to obtain a useful interpretation of the gas velocity field, we fit equation (2) to the velocity map as obtained in Section 3.1. The model fitting was done using an optimization routine that is discussed in the following section (4.3.1) allowing five of the six parameters of the model to vary freely, with the sixth $(p)$ allowed to vary in the range 1.0-1.5. Apart from the six parameters listed explicitly in equation (2), we also attempted to find the best-fitting centre coordinate $\left(x_{0}, y_{0}\right)$ of the model. The best-fitting parameters were obtained by minimizing a root-mean-square value of the residuals and are reported in Table 3. The interpretation of the results of this Bertola fit will be discussed in Section 5.1.

\subsection{Kinemetry}

The velocity model discussed above yields a best fit to the global rotation kinematics of a galaxy. It is also vital to quantify the changes in the kinematic features with radius, using methods which model the kinematics in individual rings (e.g. Begeman 1987; Jedrzejewski 1987). Such a method was generalized and established using a technique of Fourier harmonic decomposition in the software 
Table 3. Results from the kinematic analysis.

\begin{tabular}{|c|c|c|c|c|c|c|c|c|c|c|c|c|c|}
\hline Source & \multicolumn{3}{|c|}{$\begin{array}{c}\mathrm{PA}_{\mathrm{kin}} \\
\left({ }^{\circ}\right)\end{array}$} & \multicolumn{3}{|c|}{$\begin{array}{l}i_{\text {kin }} \\
\left(^{\circ}\right)\end{array}$} & \multicolumn{3}{|c|}{$\begin{array}{c}V_{\text {sys }} \\
\left(\mathrm{km} \mathrm{s}^{-1}\right)\end{array}$} & $\begin{array}{l}\Psi \\
\left({ }^{\circ}\right)\end{array}$ & $\begin{array}{c}A \\
\left(\mathrm{~km} \mathrm{~s}^{-1}\right)\end{array}$ & $\begin{array}{c}c_{0} \\
(\operatorname{arcsec})\end{array}$ & $p$ \\
\hline NGC 1386 & $88^{a}$ & 26 & 25 & 65 & 69 & 67 & 810 & 884 & 876 & 1.41 & 397 & 1.96 & 1.30 \\
\hline NGC 1667 & $165^{b}$ & 160 & 160 & 48 & 56 & 43 & 4570 & 4487 & 4479 & 4.03 & 623 & 1.67 & 1.53 \\
\hline NGC 2110 & $171^{c}$ & 171 & 178 & 39 & 51 & 63 & 2309 & 2352 & 2335 & 6.67 & 512 & 1.38 & 1.36 \\
\hline ESO $428-$ G14 & $119^{d}$ & 119 & 121 & 54 & 49 & 50 & 1752 & 1669 & 1672 & 15.23 & 376 & 4.04 & 1.33 \\
\hline NGC 3081 & $90^{e}$ & 260 & 269 & 40 & 41 & 58 & 2394 & 2385 & 2362 & 10.51 & 384 & 2.44 & 1.43 \\
\hline NGC 5728 & $30^{f}$ & 191 & 192 & 55 & 39 & 49 & 2836 & 2763 & 2763 & 20.66 & 419 & 1.17 & 1.25 \\
\hline NGC 7213 & $305^{g}$ & - & 351 & 25 & - & 42 & 1648 & - & 1783 & 70 & 174 & 0.31 & 0.95 \\
\hline
\end{tabular}

Notes. $\mathrm{PA}_{\mathrm{kin}}$ - position angle; $i_{\text {kin }}$ - inclination; $V_{\text {sys }}$ - systemic velocity. Columns (1-3): stellar kinematics from literature, median values from modKin and Bertola. Misalignment angle $(\Psi)$ obtained from the photometric PA (reported in Table 1) and the kinematic one is also shown along with other parameters $(A$, $c 0$, and $p$ ) from the Bertola model. The uncertainties of the reported values are within 5 per cent.

${ }^{a}$ Lena et al. (2015); ${ }^{b}$ Schnorr-Müller et al. (2017); ${ }^{c}$ Schnorr-Müller et al. (2014a); ${ }^{d}$ Riffel et al. (2006); ${ }^{e}$ Schnorr-Müller et al. (2016); ${ }^{f}$ Emsellem et al. (2001); ${ }^{g}$ Schnorr-Müller et al. (2014b).

Kinemetry (Krajnović et al. 2006). The method, in general, models the moment maps (velocity or higher order moments) as a series of cosine and sine terms depending on the symmetry (even) or antisymmetry (odd) of the moments. The formulation of this method is given as:

$V(a, \psi)=A_{0}(a)+\sum_{n=1}^{N} A_{n}(a) \sin (n \psi)+B_{n}(a) \cos (n \psi)$,

or in a more compact form as:

$V(a, \psi)=A_{0}(a)+\sum_{n=1}^{N} k_{n}(a) \cos \left[n\left(\psi-\phi_{n}(a)\right)\right]$,

where the length of the semimajor axis of the ellipse is denoted by $a$ and the eccentric anomaly by $\psi$. The amplitude and phase coefficients, $k_{n}$ and $\phi_{n}$, can be estimated from the following relation:

$k_{n}=\sqrt{A_{n}^{2}+B_{n}^{2}} ; \quad \phi_{n}=\arctan \left(\frac{A_{n}}{B_{n}}\right)$.

In addition to the kinematic coefficients, the code also gives the position angle $(\Gamma)$ of the kinematic major axis and the flattening parameter ( $q$; ratio of semiminor to semimajor axes of an ellipse) along the best-fitting elliptical rings. The mode of operation of the algorithm for every ellipse (given either as the number of rings or ring spacing) proceeds in a nested fashion by first extracting the kinematic profile for the first four terms $\left(A_{1}, B_{1}, A_{2}, B_{2}\right.$ in addition to $A_{0}$ ) from the Fourier expansion given in equation (3) to yield a precise estimate of the position angle and the flattening. This is due to the sensitivity of the first four coefficients to the centre coordinates, position angle, and the ellipticity $(\epsilon=1-q)$. For a visual understanding of this concept, please refer to fig. 1 in Ciambur (2015). Upon obtaining the best-fitting $(\Gamma, q)$ pair, the decomposition is repeated for the same ellipse to obtain the values of the required number of Fourier coefficients.

\subsection{Modifications to kinemetry}

Here, we elucidate the modifications that we have made in the original version of the algorithm discussed above.

(i) The version introduced by Krajnović et al. (2006) was written in the IDL programming language. We instead adopted the algorithm into MATLAB environment just for the convenience of the authors. (ii) One of the optional input parameters cover in the original version corresponds to the threshold for the ratio of the number of pixels with velocity information to the total number of pixels along a sampled ellipse. On reaching this threshold level the iteration stops without moving to the ellipse(s) placed further. Since there are cases with the availability of information beyond a certain radius, i.e. systems with multiple rings consisting of voids in between, it would be desirable to continue the iteration to the next loop instead of terminating it.

(iii) A major alteration to the original code is with regard to the eccentric anomaly. This factor corresponds to the shape of the ellipse being sampled. The original code attempts to divide an ellipse into equal bins in angle (i.e. $\phi \in[0 ; 2 \pi]$; where $\phi$ is the azimuthal angular coordinate). While this binning works well for a circle, an ellipse is relatively coarsely sampled along the semimajor axis. This effect can be negligible for many galaxies but gets more pronounced in highly inclined systems. To circumvent this problem, Ciambur (2015) introduced the following transformation:

$\psi=-\arctan \left(\frac{\tan (\phi)}{1-\epsilon}\right)$.

For details on why this formulation creates significant improvements, please see the work of Ciambur (2015).

(iv) In the original code the best-fitting parameters of the position angle and flattening were obtained by first finding the lowest value of the objective function from various pairs of $(\Gamma, q)$. The pair corresponding to the lowest value is fed to a Levenberg-Marquardt optimization routine to find a possible global minimum. We have revised this approach into a single step process by skipping the initial step of surveying using a grid. Instead we focus on obtaining the global minimum for a given bound constraint for the position angle ( $\Gamma \in[-90,90])$ and the flattening $(q \in[0,1])$. The optimization is performed using a variant of the direct search routine that is more robust to yield global results. For more details regarding the algorithm see the section below.

We refer to our modified version of the Kinemetry as modKin in the following sections. The velocity map and its associated error map ( $M_{1}$ and $M_{1, \text { err }}$ ) were used to quantify the local kinematic features in every galaxy. The velocity map was inversely weighted $\left(1 /\left(M_{1, \mathrm{err}}\right)^{2}\right)$ while solving the system of linear equations. Apart from the maps, we provided the COVER parameter of 0.50 as input along with the boundary values for the centre coordinates. The number of Fourier coefficients required from the fit was set to four to obtain the best-fitting position angle, flattening, and the centre coordinates. 
Upon obtaining these values, we ran the modKin code again at these fixed values for 10 Fourier terms. In all cases, the number of ellipses to be fit was initially set to 100 with a ring spacing set according to the maximum size of the interferometric beam.

\subsubsection{Optimization algorithm}

The two different models (Bertola and Kinemetry) that are discussed above rely heavily on the use of an optimization algorithm to obtain the best-fitting values. Since both models are non-linear, it is essential to implement a routine that effectively explores the parameter space to converge to a global minimum. We, therefore, adopt a variant of the direct search method, which is a derivativefree optimization strategy that is becoming quite indispensable in practice (Audet \& Hare 2017).

The mesh adaptive direct search (MADS) is one such method that generates meshes of the parameter space (Audet \& Dennis 2006). It then performs an adaptive search on the generated trial points as per the tuning parameters, which controls the effectiveness of the exploration, to minimize the given objective function. The non-linear optimization coupled with the MADS algorithm guarantees a much faster convergence on the global minimum (Le Digabel 2011). We therefore use the $\mathrm{MADS}^{1}$ algorithm for all our analysis.

The choice of the dimensionality is one of the primary concerns in choosing any optimization routine. We tested the adopted optimization algorithm with the Bertola model and modKin code on 1000 different simulated velocity maps with known sets of parameters. In all our simulations, the optimization routine was able to recover the parameters to within an error of 1 per cent. We, however, caution the reader that this effectiveness is guaranteed only for the lower dimensionality, i.e. we have a maximum of eight parameters to fit in a given model in this work. For models with more parameters, rigorous analysis with the optimization routine will be required.

\subsection{D-modelling algorithms}

Algorithms for modelling the spectral cube directly exist in literature. These packages have the advantage of taking into the account the systematics from the observations effectively. Some of the wellknown packages are GalPaK (Bouché et al. 2015), ${ }^{3 \mathrm{D}} \mathrm{BAROLO}$ (Di Teodoro \& Fraternali 2015), and TiRiFiC (Józsa et al. 2007). In the ALMA and IFU era where the resolution plays a major role it is essential to start implementing these 3D approaches over the $2 \mathrm{D}$ ones. However, for this work, we only consider the 2D modelling approach owing to the lack of features in the mentioned 3D packages to constrain or demarcate the non-axisymmetric features effectively.

\section{RESULTS}

\subsection{Kinematic centres}

In our attempt to model the kinematics of all the galaxies, we also attempted to constrain their kinematic centre. The models of all the galaxies and their residuals are shown in Fig. 2 and Appendix C (online), which is provided online. For both our models, we searched for the best-fitting kinematic centre within $5 \operatorname{arcsec}$ of the continuum

\footnotetext{
${ }^{1}$ https://www.gerad.ca/nomad/Project/Home.html
}

peak position. The result obtained from both the models seem to agree within a few $( \pm 5)$ pixels. We decided to use the result from the modKin code for further interpretation. The centre coordinates were compared to those given in 2MASS (Table 4). For robustness, we consider the centre coordinates as a new estimate only if the difference in value is larger than the synthesized beam major axis. We, thus, were able to obtain new kinematic centres for NGC 1667 and NGC 5728. Interestingly, both these sources have a peculiar nuclear morphology as can be inferred from their CO moment 0 maps and a comparison of these with the HST images. NGC 1667 has two dominant CO-bright peaks in the nuclear region, separated by $\sim 2 \operatorname{arcsec}$ (Fig. B1; online). The new kinematic centre is coincident with the western CO-bright component while the 2MASS centre position is midway between the two CO-bright peaks. NGC 5728 is also characterized by two CO-bright components on the NE and SW part of the nucleus, separated by $\sim 2$ arcsec. This galaxy is a candidate for a double rotating core (Son et al. 2009). In the HST maps (Fig. B5; online), the offset of the nucleus is quite evident, which could be a possible effect of a merger.

\subsection{Local kinematic results}

The radial dependence of the position angle $(\Gamma)$, inclination $(\theta)$, and the amplitude terms obtained from modKin are vital in constraining the kinematic features in the velocity maps. The profiles for all the galaxies except for NGC 7213 (was impossible to obtain meaningful fits from modKin owing to the sparse information) are shown in the Fig. 3 and Appendix C (online). The radial profiles can be interpreted as follows:

(i) The position angle in most galaxies shows a smooth evolution with occasional variations, with the exception of NGC 5728 (Fig. C10; online) which remains relatively constant for the most part, while a change of $\approx 160^{\circ}$ is noticed in NGC 1667 (Fig. C2; online).

(ii) The change in inclination with radius of the galaxies ESO 428-G14 (Fig. C6; online), NGC 1386 (Fig. 3), and NGC 1667 (Fig. C2; online) seem relatively smooth; the occasional sudden fluctuations is most likely attributable to noise.

(iii) The asymmetric circular velocity component (traced by the $B_{1} \sin \theta$; hereafter denoted as $V_{\text {rot }}$ ) and the $k_{1}$ amplitude term are both plotted in the figures so as to identify the galaxies with a departure from a pure circular rotation. Of the listed sources, NGC 1667 (Fig. C2; online) and NGC 5728 (Fig. C10; online) are the only ones that show a deviation from a circular rotation within the inner 2 arcsec. Otherwise, most galaxies show a smooth increase in $V_{\text {rot }}$ which flattens at around 2 arcsec.

(iv) There is growing evidence for power in even Fourier coefficients, despite the common practice of considering only the odd kinematic coefficients for the Moment 1 map, which could provide more clues to the perturbations in a galaxy. Hence, we show the amplitudes of terms $k_{2}, k_{3}, k_{4}$, and $k_{5}$ (normalized to the dominant term $k_{1}$ term). All galaxies show stark variations in all four amplitude parameters. This is discussed further in Section 6.

In works of SAURON (Krajnović et al. 2008) and ATLAS ${ }^{3 D}$ (Krajnović et al. 2011) surveys which studied the stellar kinematics in a large sample of galaxies, a classification scheme based on the profiles exhibited by parameters like the position angle, inclination, and the amplitude terms of the Fourier coefficients was introduced. All galaxies analysed here reveal multiple characteristics, such as a deviation greater than tens of degrees around the median value 

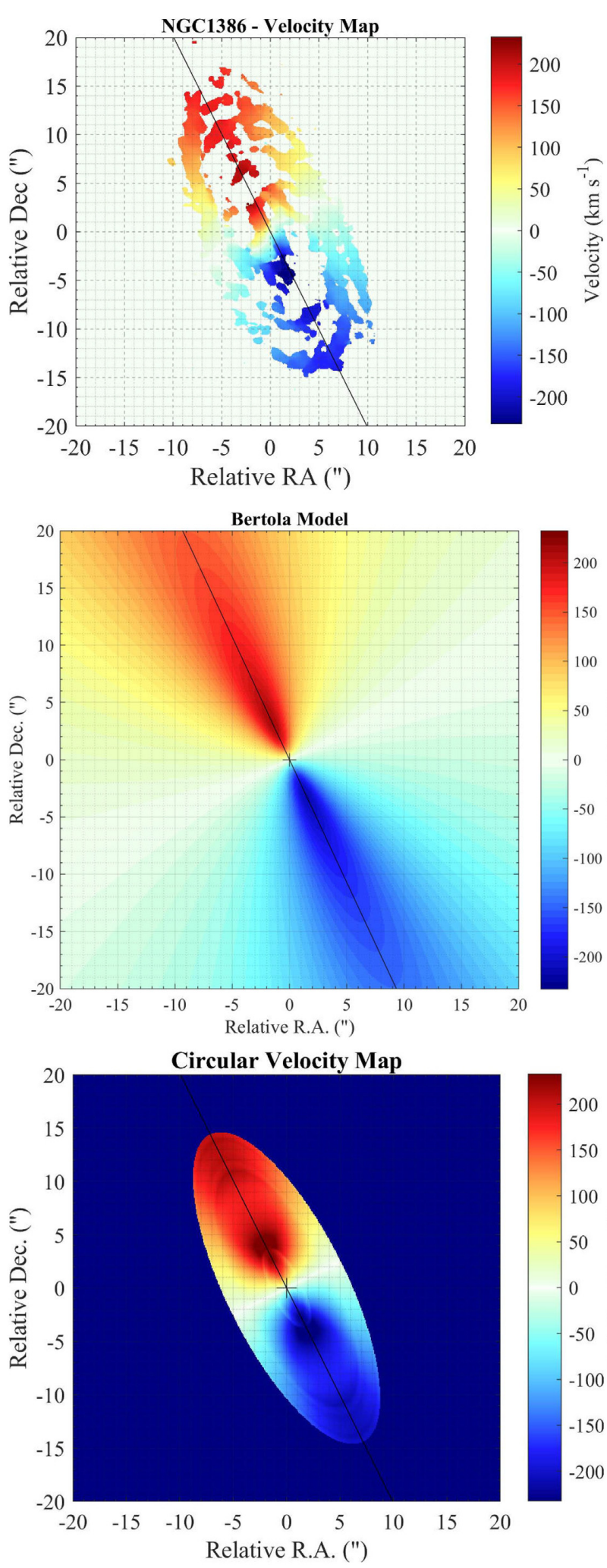

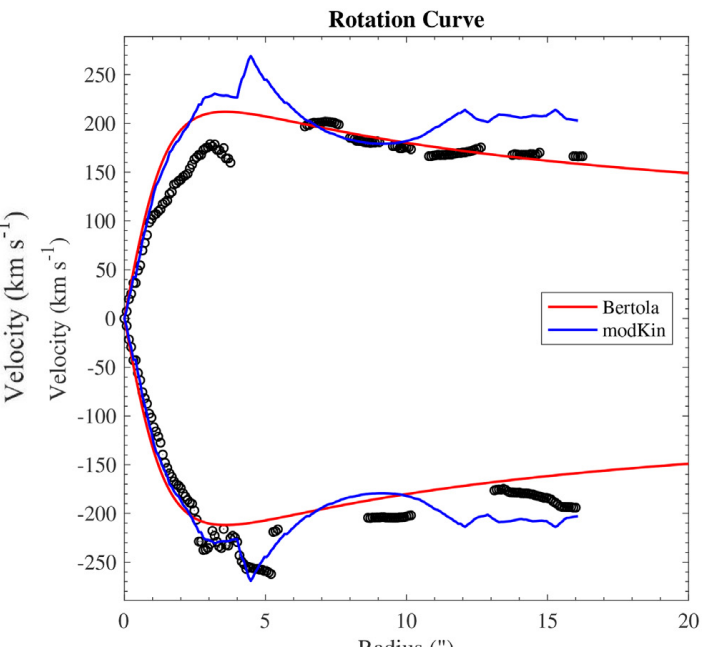

Bertola Residual Map
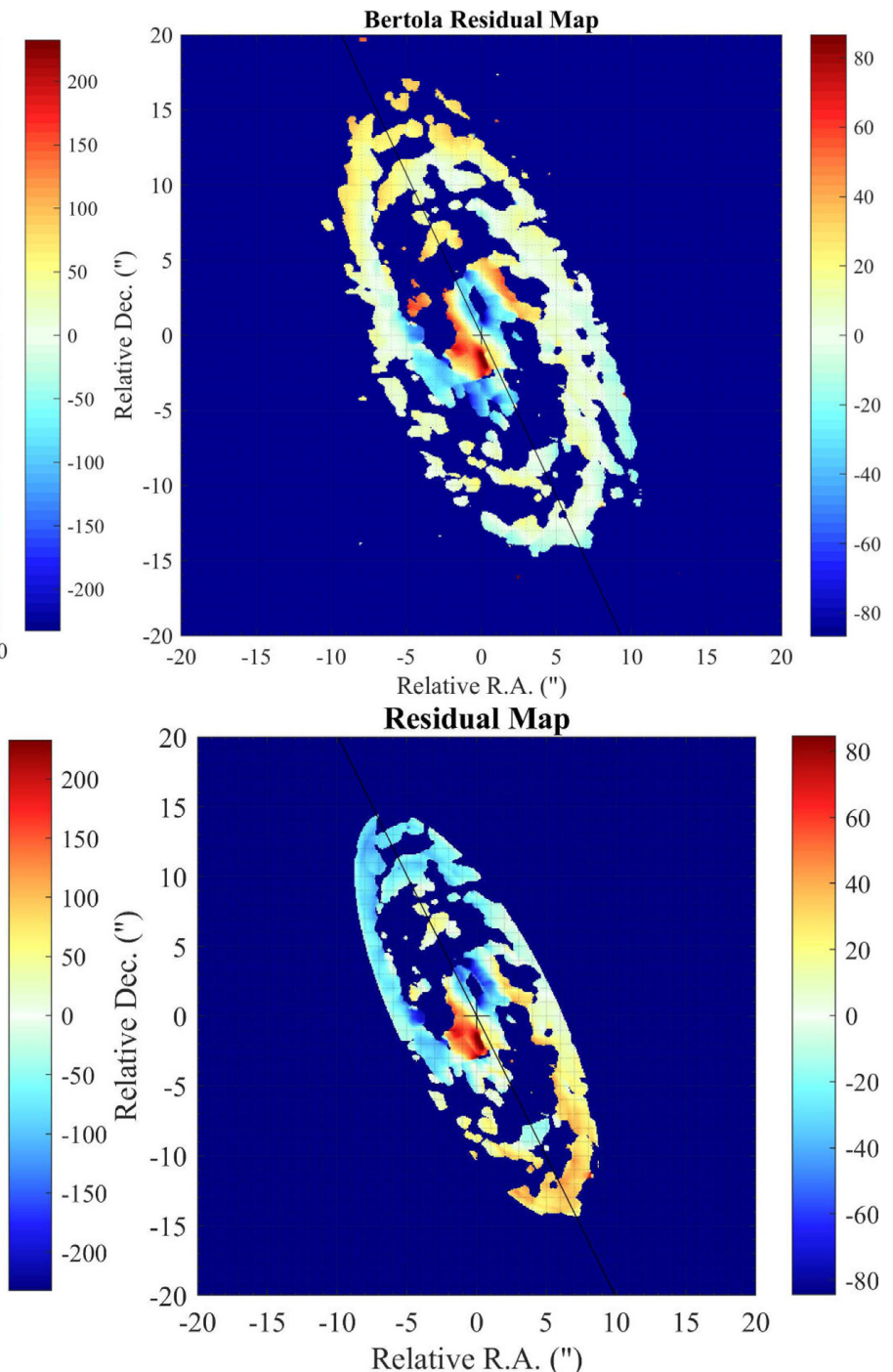

Residual Map

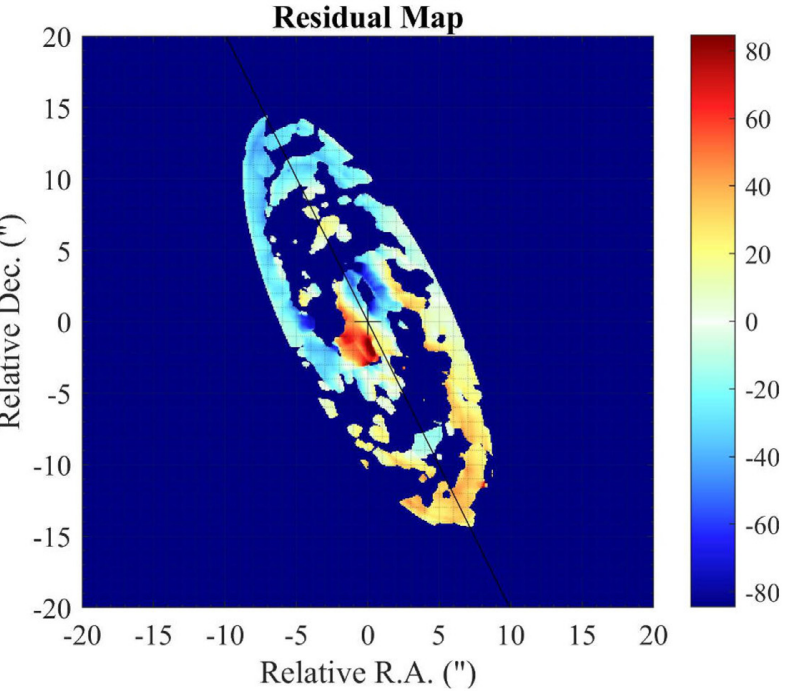

Figure 2. NGC 1386. Top-row: ALMA velocity map (left) and rotation curves (right). Middle-row: Bertola rotation model (left) and the corresponding residual map (right). Bottom-row: Circular velocity model generated from the $B_{1} \cos \theta$ term of the modKin package (left) and the residual map (right). The solid black line in all the images is the kinematic major axis. The black open circles in the rotation curve correspond to the data while the red and the blue lines correspond to the rotation curve from Bertola and modKin models that are shown in the left-hand panel of the middle and bottom row. 
Table 4. New centre coordinates.

\begin{tabular}{lccc}
\hline Sources & RA & Dec. & $\begin{array}{c}\text { Separation } \\
\text { (arcsec) }\end{array}$ \\
\hline NGC 1667 & $04: 48: 37.14$ & $-06: 19: 11.56$ & 0.92 \\
NGC 5728 & $14: 42: 23.87$ & $-17: 15: 10.93$ & 0.94 \\
\hline
\end{tabular}

in position angle and inclination along with rapid variations in amplitude of most Fourier components. We adopt the same system as in the aforementioned works here to classify the galaxies as follows:

(i) Kinematic twist: Variation in position angle $>10^{\circ}$ around the median value. All sources with the exception of NGC 5728 and NGC 7213 fall into this category. These two galaxies show a smooth progression of the velocity as reflected by minor deviations of the position angle around the median value.

(ii) Kinematically distinct core: Abrupt variation of $>20^{\circ}$ in the position angle of adjacent rings along with the decline of the term $k_{1}$ to zero. In most cases, the ratio $k_{5} / k_{1}$ shows a peak around this region. NGC 1386 (Fig. 3) is the only candidate that belongs to this class. The position angle of the source drops by $\sim 20^{\circ}$ at $4 \operatorname{arcsec}$ $(220 \mathrm{pc})$ from the nucleus. Although $k_{1}$ does not show a significant decline, the $k_{5} / k_{1}$ term peaks around this radius.

(iii) Counter-rotating core: This is a unique case of the above category where the change in the position angle is of the order of $180^{\circ}$. NGC 1667 (Fig. C2; online) exhibits such a property with a change of about $150^{\circ}$ in the inner $2 \operatorname{arcsec}(634 \mathrm{pc})$ in addition to the significant drop in the rotational velocity term and a peak in $k_{5} / k_{1}$. The origin of such systems could be a result of a merger or an inflow of intergalactic clouds, in turn spurring the nuclear activity.

NGC 5728 (Fig. C10; online) shows almost a constant phase in position angle. However, its inclination drops significantly $\left(\sim 80^{\circ}\right)$ in the inner 2-3 arcsec (428-642 pc) followed by a smooth increase. This trait and the decline of the rotational velocity in the same region favours the double rotating core hypothesis proposed by Son et al. (2009). This feature will be explored in a subsequent work. We have restricted our discussion here to results obtained from considering only odd moments in the modKin fits and note that any signatures such as those of possible mergers could have been constrained by modelling the even moments.
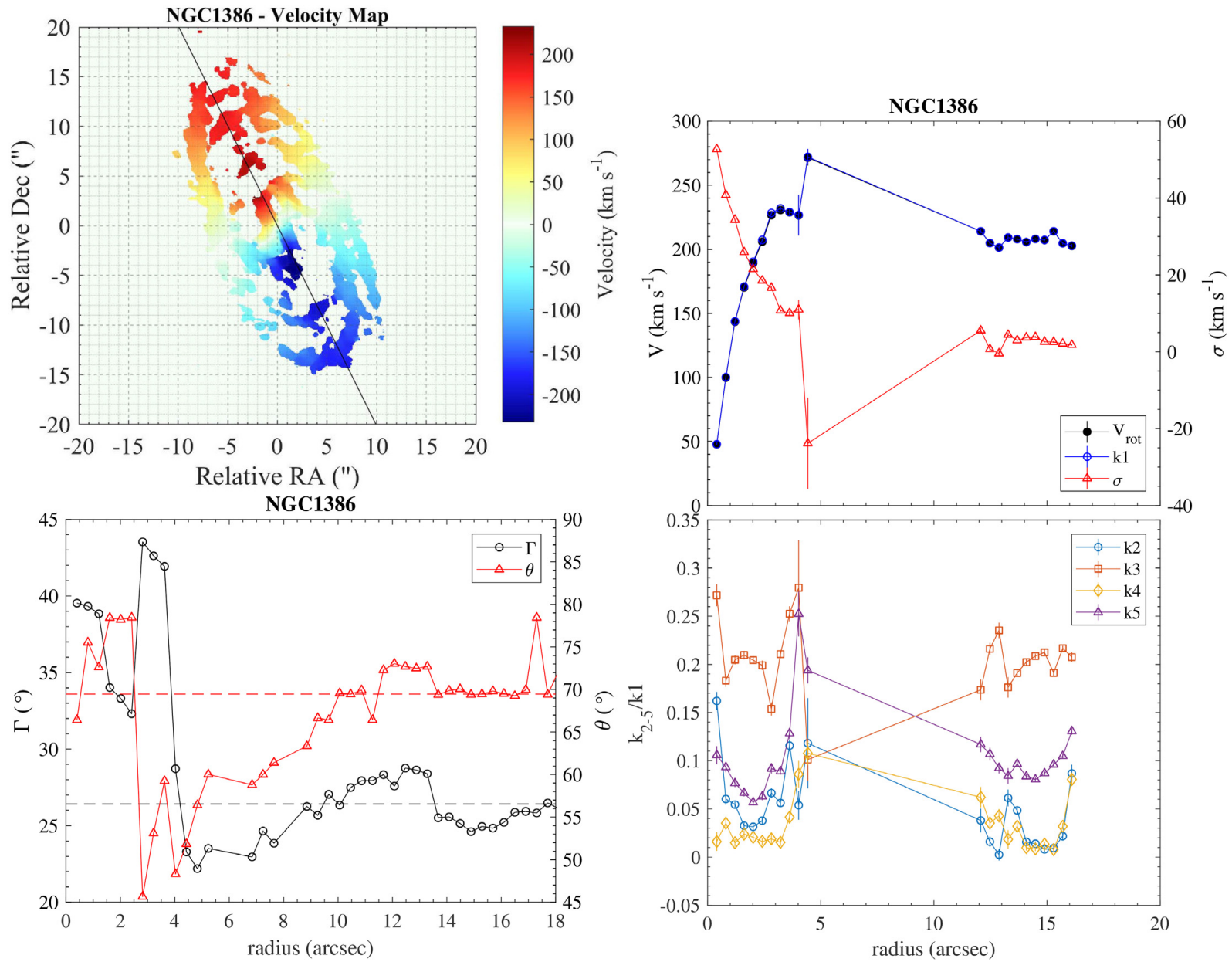

Figure 3. NGC 1386. Top-row: ALMA velocity map (left) and the velocity and velocity dispersion profile (right). The axis of the rotational velocity term $V_{\text {rot }}$ and the Fourier amplitude term $k_{1}$ corresponds to the left ordinate while that for the velocity dispersion term $(\sigma)$ corresponds to the right. Bottom-row: Profile of the position angle and inclination along with their respective median values denoted by the dashed line (left) and the amplitudes of the Fourier coefficients (right). The left ordinate corresponds to the position angle and the right one to the inclination. All the amplitude of the Fourier coefficients that are plotted are normalized to the first term $k_{1}$. 


\subsection{Global results}

To understand the global kinematics of the galaxies we modelled the velocity maps using the Bertola model (equation 2), which provides the maximum velocity, slope of the rotation curve after reaching its maximum and the parameter $c_{0}$, that gives the radius at which the velocity reaches 70 per cent of its maximum amplitude. This is obtained in addition to the position angle, inclination, and the systemic velocity of the galaxy. The maximum velocity given by this model varies by $\sim 450 \mathrm{~km} \mathrm{~s}^{-1}$ across the galaxies. The slope of NGC 7213 is at the lower end (probably due to the sparsity in the data) while NGC 1667 occupies the upper bound. The slope of the remaining galaxies fall in the range 1.2-1.4 (last column in Table 3 ). The region with the 70 per cent velocity in most systems are in the inner 1-2 arcsec with the exception of NGC 3081 (at 2.4 arcsec otherwise $463.2 \mathrm{pc}$ ) and ESO $428-\mathrm{G} 14$ (at 4 arcsec otherwise $536 \mathrm{pc}$ ).

We compare the median values of the parameters - position angle, inclination, and systemic velocity - obtained from the modKin code with the Bertola model. These estimates are reported in columns 2 and 3 of Table 3 . The position angle of all sources seems to comply within $\sim 10^{\circ}$ of one another and the systemic velocity as well within $\sim 20 \mathrm{~km} \mathrm{~s}^{-1}$. Inclination seems to be the one with a relatively larger discrepancy, reaching as high as $\sim 16^{\circ}$. In systems like ESO 428-G14 and NGC 1386, all three estimates seem to agree with each other. This inference does raise a claim for a disclike rotation of these galaxies, which is indeed a favourable postulate from a visual inspection of their velocity maps.

The models under scrutiny in this work does generate a map of its own. Every such map is subtracted from the original velocity map of the galaxy to produce the residual, which provides information on the non-circular motion arising from various perturbations. The model used for this purpose in the case of modKin is the one that is obtained from just the circular velocity term, therefore making the direct comparison of the residuals from both the models feasible. These models are shown in Fig. 2 and Appendix C (online). Amongst all the galaxies, the residual maps of NGC 5728 (Fig. C9; online) are the only ones that are similar for the two models. In galaxies NGC 1667 (Fig. C1; online), NGC 2110 (Fig. C3; online), and NGC 3081 (Fig. C7; online), the residuals reveal different possible perturbations. Finally, in ESO 428-G14 (Fig. C5; online) the residual maps in the outer region (beyond 3 arcsec otherwise $>402$ pc) and those in NGC 1386 (Fig. 3) within the inner $3 \operatorname{arcsec}(<165 \mathrm{pc})$ seem to be in agreement. The residual map of NGC 7213 (Fig. C11; online) generated using the Bertola model reveals some perturbations in the inner 5 arcsec (550 pc).

Apart from the maps mentioned above, we also extracted the rotation curves of all the galaxies along the major axis as given by the median of the position angle from the code modKin. The rotation curves are plotted after the subtraction of the systemic velocity. Overplotted on every curve are those extracted from the two different models. NGC 5728 (Fig. C9; online) is the only galaxy with a rotation curve suggesting a symmetric rotation as also supported by the models; NGC 1386 (Fig. 2) seems to indicate a symmetric rotation for the most part with minor deviations in the inner $5 \operatorname{arcsec}(275 \mathrm{pc})$. The observed rotation of the rest of the galaxies is either highly asymmetric or show strong deviations from the symmetry at various radii from the centre. No conclusion can be invoked from the rotation curve of NGC 7213.

There is clear evidence for the presence of non-axisymmetric features in certain galaxies, which for example, in NGC 1386 was also proven by CO (1-0) observations of Zabel et al. (2019). We, however, caution the reader that the results reported in this work are primarily based on the interpretation of the intensity weighted mean velocity maps. As a result, we do not discuss non-axisymmetric features here; instead, these form the bulk of the discussion in a subsequent work (Ramakrishnan et al. in preparation).

\subsection{Misalignment of photometric and kinematic axis}

Following Franx, Illingworth \& de Zeeuw (1991), we compute the misalignment angle between the photometric and kinematic position angle as given by the following relation:

$\sin \Psi=\left|\sin \left(\mathrm{PA}_{\text {phot }}-\mathrm{PA}_{\text {kin }}\right)\right|$.

The photometric position angle obtained from the 2MASS (Table 1) is used in conjunction with the kinematic position angle reported in column 3 of Table 3 . The misalignment angle thus obtained lies in the range $0-90^{\circ}$, regardless of the $180^{\circ}$ ambiguity between the two position angles. The estimated values are reported in Table 3. We classify all galaxies with $\Psi>10^{\circ}$ as being misaligned. This makes ESO 428-G14, NGC 3081, NGC 5728, and NGC 7213 as misaligned candidates.

\section{DISCUSSION AND SUMMARY}

The correspondence between the molecular and ionized gas kinematics has been explored in earlier works (e.g. Wong, Blitz \& Bosma 2004; Davis et al. 2013; de Blok et al. 2016) showing a good agreement in a majority of cases. In works by the ATLAS ${ }^{3 \mathrm{D}}$ group, it was shown that the rotation velocities of the ionized gas to be much lower than that of the molecular ones. The circular rotation velocities obtained from the cold molecular gas are in general a better estimate over the stellar ones.

The close correspondence between the dust and the molecular gas of the seven galaxies can be inferred from the overlay of the moment 0 maps on the HST ones. This correspondence implies that the internal stellar mass-loss is the primary factor for the production of the molecular gas.

All galaxies except NGC 5728 were already explored using the GEMINI IFU observations by our group (Riffel et al. 2006; SchnorrMüller et al. 2014a,b, 2016, 2017; Lena et al. 2015). Throughout our study, we found the position angle of most galaxies, with the exception of NGC 1386, to be in agreement with those reported from the stellar kinematics (Table 3). The inclination and systemic velocity, however, seem to show a significant discrepancy. This disparity can be reconciled by an external origin scenario for the gas in such systems.

The local kinematics of the galaxies show trademarks of multicomponent systems. They were categorized into systems with kinematic twists, those with decoupled and a counter-rotating core. This classification already implies the prevalence of perturbations in the nuclear regions. The various perturbations that could play a vital role in these systems are outflow/inflow of gas, warping of spiral arms, barred (possibly multi-barred) systems and minor mergers, among others. Through the ionized gas kinematics, our group had already studied the outflows and inflows in various systems reported here. It is important to understand the effect of non-circular motions in these systems. The presence of such motions can be deciphered from the residual maps of various galaxies. The non-circular motions explain the dynamical processes in play and the transport of gas responsible under various phenomena under extreme conditions. The later scenario can be further understood by the fuelling processes that are governed either by external accretion 
or through secular process. We defer to the study of these motions and its connection to the ionized gas kinematics to a subsequent work (Ramakrishnan et al., in preparation).

We thus summarize our findings from this work as follows:

(i) The CO integrated maps show spiral arms structure within the inner hundreds of parsecs with disc-like rotation.

(ii) The continuum emission from three sources are point like while the other three show an extension varying from 3 to 6 arcsec. NGC 1667 is the only source with no continuum emission above the noise level.

(iii) The local kinematics of the galaxies are explored using a modified version of the package Kinemetry. Based on the radial profiles of the position angle, inclination, and various amplitude terms, we are able to identify all galaxies as multicomponent systems. They are further classified into those exhibiting a kinematic twist, with a decoupled core and also into a counter-rotating one. NGC 1667 is identified to fall into the latter category with the core counter-rotating by about $150^{\circ}$. The severity seen in the inclination profile of NGC 5728 seems to be an effect of two distinct cores as reported in Son et al. (2009).

(iv) The global kinematics are studied using the Bertola model, in addition to the median values of the local profile. The position angle and the systemic velocity agree within the errors, while the inclination shows a discrepancy. Using the global values, the misalignment angle is also computed showing that four out of seven galaxies to be misaligned with the photometric major axis.

(v) The centre position of two sources NGC 1667 and NGC 5728 are revised to a new estimate based on the kinematics. Both these sources are equally good candidates for a system with an offset nuclei.

(vi) The residual maps of most galaxies show the presence of perturbations with clear signs of outflows/inflows in most systems. This will be a subject of future work.

\section{ACKNOWLEDGEMENTS}

We thank the anonymous referee for valuable comments that improved this manuscript. VR acknowledges the support from the ALMA CONICYT project 31140007, 22930.921.02 Prog. Financ. BASAL-PFB/06 and Anillo ACT 172033. NN acknowledges support from BASAL-PFB/06 and extension, Fondecyt 1171506, and Anillo ACT 172033. RAR acknowledges support from CNPq and FAPERGS. This paper makes use of the following ALMA data: ADS/JAO.ALMA\#2012.1.00474.S and 2015.1.00086.S. ALMA is a partnership of ESO (representing its member states), NSF (USA) and NINS (Japan), together with NRC (Canada), MOST and ASIAA (Taiwan), and KASI (Republic of Korea), in cooperation with the Republic of Chile. The Joint ALMA Observatory is operated by ESO, AUI/NRAO and NAOJ. This research used the facilities of the Canadian Astronomy Data Centre operated by the National Research Council of Canada with the support of the Canadian Space Agency. This paper uses data taken with the NASA/ESA Hubble Space Telescope, obtained from the Data Archive at the Canadian Astronomy Data Centre. The Hubble Space Telescope is a collaboration between the Space Telescope Science Institute (STScI/NASA), the Space Telescope European Coordinating Facility (ST-ECF/ESA), and the Canadian Astronomy Data Centre (CADC/NRC/CSA). Use was also made of the NASA/IPAC Extragalactic Database (NED), which is operated by the Jet Propulsion Laboratory, California Institute of Technology, under contract with
NASA. This research has made use of NASA's Astrophysics Data System. This research has made use of the SIMBAD database, operated at CDS, Strasbourg, France.

\section{REFERENCES}

Aalto S., Muller S., Sakamoto K., Gallagher J. S., Martín S., Costagliola F., 2012, A\&A, 546, A68

Alatalo K. et al., 2011, ApJ, 735, 88

Alatalo K. et al., 2013, MNRAS, 432, 1796

Audet C., Hare W., 2017, Derivative-Free and Blackbox Optimization. Springer Series in Operations Research and Financial Engineering. Springer International Publishing, Cham

Audet C., Dennis J., Jr., 2006, SIAM J. Optim., 17, 188

Begeman K. G., 1987, PhD thesis. Kapteyn Institute

Bertola F., Bettoni D., Danziger J., Sadler E., Sparke L., de Zeeuw T., 1991, ApJ, 373, 369

Blank D. L., Harnett J. I., Jones P. A., 2005, MNRAS, 356, 734

Bolatto A. D. et al., 2017, ApJ, 846, 159

Bouché N., Carfantan H., Schroetter I., Michel-Dansac L., Contini T., 2015, AJ, 150, 92

Buta R., 1990, ApJ, 351, 62

Buta R., Purcell G. B., 1998, AJ, 115, 484

Cappellari M. et al., 2011, MNRAS, 413, 813

Ciambur B. C., 2015, ApJ, 810, 120

Cicone C. et al., 2014, A\&A, 562, A21

Combes F. et al., 2013, A\&A, 558, A124

Combes F. et al., 2014, A\&A, 565, A97

Crenshaw D. M., Kraemer S. B., Gabel J. R., 2003, AJ, 126, 1690

Davis T. A. et al., 2013, MNRAS, 429, 534

de Blok W. J. G. et al., 2016, AJ, 152, 51

de Vaucouleurs G., de Vaucouleurs A., Corwin H. G., Jr., Buta R. J., Paturel G., Fouqué P., 1991, Third Reference Catalogue of Bright Galaxies. Volume I: Explanations and references. Volume II: Data for galaxies between $0 h$ and $12^{h}$. Volume III: Data for galaxies between $12^{h}$ and $24^{h}$. Springer-Verlag, New York

de Zeeuw P. T. et al., 2002, MNRAS, 329, 513

Di Matteo T., Springel V., Hernquist L., 2005, Nature, 433, 604

Di Teodoro E. M., Fraternali F., 2015, MNRAS, 451, 3021

Diniz M. R., Riffel R. A., Storchi-Bergmann T., Winge C., 2015, MNRAS, 453, 1727

Emsellem E., Greusard D., Combes F., Friedli D., Leon S., Pécontal E., Wozniak H., 2001, A\&A, 368, 52

Falcke H., Wilson A. S., Simpson C., Bower G. A., 1996, ApJ, 470, L31

Falcke H., Wilson A. S., Simpson C., 1998, ApJ, 502, 199

Fathi K., van de Ven G., Peletier R. F., Emsellem E., Falcón-Barroso J., Cappellari M., de Zeeuw T., 2005, MNRAS, 364, 773

Ferrarese L., Merritt D., 2000, ApJ, 539, L9

Ferruit P., Wilson A. S., Mulchaey J., 2000, ApJS, 128, 139

Finlez C., Nagar N. M., Storchi-Bergmann T., Schnorr-Müller A., Riffel R. A., Lena D., Mundell C. G., Elvis M. S., 2018, MNRAS, 479, 3892

Franx M., Illingworth G., de Zeeuw T., 1991, ApJ, 383, 112

García-Burillo S. et al., 2003, A\&A, 407, 485

Gebhardt K. et al., 2000, ApJ, 539, L13

Gültekin K. et al., 2009, ApJ, 698, 198

Hopkins P. F., Quataert E., 2010, MNRAS, 407, 1529

Hopkins P. F., Hernquist L., Cox T. J., Kereš D., 2008, ApJS, 175, 356

Humire P. K. et al., 2018, A\&A, 614, A94

Jedrzejewski R. I., 1987, MNRAS, 226, 747

Józsa G. I. G., Kenn F., Klein U., Oosterloo T. A., 2007, A\&A, 468, 731

Kauffmann G., Haehnelt M., 2000, MNRAS, 311, 576

Kormendy J., Kennicutt R. C., Jr., 2004, ARA\&A, 42, 603

Krajnović D. et al., 2008, MNRAS, 390, 93

Krajnović D. et al., 2011, MNRAS, 414, 2923

Krajnović D., Cappellari M., de Zeeuw P. T., Copin Y., 2006, MNRAS, 366, 787 
Lena D. et al., 2015, ApJ, 806, 84

Levy R. C. et al., 2018, ApJ, 860, 92

Le Digabel S., 2011, ACM Trans. Math. Softw., 37, 1

Maciejewski W., 2004, MNRAS, 354, 892

Maciejewski W., Teuben P. J., Sparke L. S., Stone J. M., 2002, MNRAS, 329,502

Malkan M. A., Gorjian V., Tam R., 1998, ApJS, 117, 25

Mazzuca L. M., Knapen J. H., Veilleux S., Regan M. W., 2008, ApJS, 174, 337

McMullin J. P., Waters B., Schiebel D., Young W., Golap K., 2007, in Shaw R. A., Hill F., Bell D. J., eds, ASP Conf. Ser. Vol. 376, Astronomical Data Analysis Software and Systems XVI. Astron. Soc. Pac., San Francisco, p. 127

Morganti R., Tadhunter C. N., Oosterloo T. A., 2005, A\&A, 444, L9

Morganti R., Oosterloo T., Oonk J. B. R., Frieswijk W., Tadhunter C., 2015, A\&A, 580, A1

Morganti R., Veilleux S., Oosterloo T., Teng S. H., Rupke D., 2016, A\&A, 593, A30

Mulchaey J. S., Wilson A. S., Bower G. A., Heckman T. M., Krolik J. H., Miley G. K., 1994, ApJ, 433, 625

Mundell C. G., Wilson A. S., Ulvestad J. S., Roy A. L., 2000, ApJ, 529, 816

Mundell C. G., Ferruit P., Nagar N., Wilson A. S., 2009, ApJ, 703, 802

Nagar N. M., Wilson A. S., Mulchaey J. S., Gallimore J. F., 1999, ApJS, 120, 209

Planck Collaboration XIII, 2016, A\&A, 594, A13

Quinn P. J., Hernquist L., Fullagar D. P., 1993, ApJ, 403, 74

Riffel R. A., Storchi-Bergmann T., Winge C., Barbosa F. K. B., 2006, MNRAS, 373, 2

Riffel R. A., Storchi-Bergmann T., Winge C., McGregor P. J., Beck T., Schmitt H., 2008, MNRAS, 385, 1129

Riffel R. A., Storchi-Bergmann T., Winge C., 2013, MNRAS, 430, 2249

Rubin V. C., 1980, ApJ, 238, 808

Sánchez S. F. et al., 2012, A\&A, 538, A8

Sanders D. B., Soifer B. T., Elias J. H., Madore B. F., Matthews K., Neugebauer G., Scoville N. Z., 1988, ApJ, 325, 74

Schmitt H. R., Ulvestad J. S., Antonucci R. R. J., Kinney A. L., 2001, ApJS, 132,199

Schmitt H. R., Calzetti D., Armus L., Giavalisco M., Heckman T. M., Kennicutt R. C., Jr., Leitherer C., Meurer G. R., 2006, ApJS, 164, 52

Schnorr-Müller A., Storchi-Bergmann T., Nagar N. M., Robinson A., Lena D., Riffel R. A., Couto G. S., 2014a, MNRAS, 437, 1708

Schnorr-Müller A., Storchi-Bergmann T., Nagar N. M., Ferrari F., 2014b, MNRAS, 438, 3322

Schnorr-Müller A., Storchi-Bergmann T., Robinson A., Lena D., Nagar N. M., 2016, MNRAS, 457, 972

Schnorr-Müller A., Storchi-Bergmann T., Ferrari F., Nagar N. M., 2017, MNRAS, 466, 4370

Schommer R. A., Caldwell N., Wilson A. S., Baldwin J. A., Phillips M. M., Williams T. B., Turtle A. J., 1988, ApJ, 324, 154

Simões Lopes R. D., Storchi-Bergmann T., de Fátima Saraiva M., Martini P., 2007, ApJ, 655, 718

Skrutskie M. F. et al., 2006, AJ, 131, 1163 (2MASS)

Slater R. et al., 2019, A\&A, 621, A83

Son D.-H., Hyung S., Ferruit P., Pécontal E., Lee W.-B., 2009, MNRAS, 395, 692

Storchi-Bergmann T., Schnorr-Müller A., 2019, Nat. Astron., 3, 48

Storchi-Bergmann T., Rodriguez-Ardila A., Schmitt H. R., Wilson A. S., Baldwin J. A., 1996, ApJ, 472, 83

Tadhunter C., Morganti R., Rose M., Oonk J. B. R., Oosterloo T., 2014, Nature, 511, 440

Tremaine S. et al., 2002, ApJ, 574, 740

Weaver K. A., Wilson A. S., Baldwin J. A., 1991, ApJ, 366, 50

Wilson A. S., Braatz J. A., Heckman T. M., Krolik J. H., Miley G. K., 1993, ApJ, 419, L61

Wong T., Blitz L., Bosma A., 2004, ApJ, 605, 183

Young L. M. et al., 2011, MNRAS, 414, 940

Zabel N. et al., 2019, MNRAS, 483, 2251

\section{SUPPORTING INFORMATION}

Supplementary data are available at MNRAS online.

Appendix B. HST and ALMA images of galaxies Appendix C. Results from kinematic studies

Please note: Oxford University Press is not responsible for the content or functionality of any supporting materials supplied by the authors. Any queries (other than missing material) should be directed to the corresponding author for the article.

\section{APPENDIX A: NOTES ON INDIVIDUAL SOURCES}

NGC 1386 is an Sb/c galaxy with a Seyfert 2 nucleus (Malkan et al. 1998). The HST imaging of this galaxy by Ferruit, Wilson \& Mulchaey (2000) reveals dusty nuclear region running parallel to the major axis. The ellipticity of the disc from the nucleus and outward increases from about 0.25 to 0.5 at $\sim 1.5 \mathrm{kpc}$ with signs of warping. The radio observations of the source reveal a compact core with a jet that extends southward (Nagar et al. 1999). Although a similar extension was reported in [O III] and $\mathrm{H} \alpha+[\mathrm{N} \mathrm{II}]$ maps (Weaver, Wilson \& Baldwin 1991; Storchi-Bergmann et al. 1996; Ferruit et al. 2000), no clear correspondence between the optical and radio was inferred (Mundell et al. 2009). The integral field spectroscopic observations in the optical by Lena et al. (2015) of the central $\sim 500 \mathrm{pc}$ reveal a bright nuclear component with two lobes extending $\sim 200 \mathrm{pc}$ north and south of the nucleus. Two kinematic features with velocity dispersions of $\approx 90$ (entire field of view) and $\approx 200 \mathrm{~km} \mathrm{~s}^{-1}$ (inner $150 \mathrm{pc}$ ) were reported by the authors.

NGC 1667 is a low-luminosity Sc galaxy with Seyfert 2 nucleus (Malkan et al. 1998). The galaxy is characterized by multiple spiral arms with dust contamination in the central kpc (Schmitt et al. 2006). Two roughly symmetrical spiral arms are visible in the nuclear region. The ultraviolet to IR observations of the galaxy by Schmitt et al. (2006) reveal that only a minor fraction of the UV emission originates from the nucleus unlike the $\mathrm{H} \alpha$ emission which is significantly stronger. Both the $\mathrm{UV}$ and $\mathrm{H} \alpha$ emission arise from the star-forming region along the spiral arms. The nuclear radio emission of the galaxy at $8.4 \mathrm{GHz}$ is weak $(\sim 7.5 \mathrm{mJy})$ with no distinct features but a diffuse emission around the galaxy. The optical IFU observations by Schnorr-Müller et al. (2017) reveal complex morphology with two kinematic components of $\sigma \approx 200$ and $\sigma \approx 400 \mathrm{~km} \mathrm{~s}^{-1}$ over the entire field of view and the inner 2 arcsec, respectively.

$N G C 2110$ is a well-known Sa Seyfert 2 galaxy (Malkan et al. 1998). The HST [O III] and $\mathrm{H} \alpha+[\mathrm{N}$ II] emission line images of the galaxy is characterized by a dusty circumnuclear disc and a long S-shaped jet (Mulchaey et al. 1994). A similar jetlike feature was reported by Nagar et al. (1999) that extends for about 4 arcsec along the north-south direction. Despite the prevalence of the extended emission along a similar position angle at both optical and radio wavebands, the emission regions are not causally related. The peak of the radio emission is off-centred with no corresponding line emission. Schnorr-Müller et al. (2014a) studied the feedback processes of the galaxy using the Gemini-IFU observations. The authors report four kinematic components of velocity dispersions in the range $60-220 \mathrm{~km} \mathrm{~s}^{-1}$ for three of them, and a nuclear component in $220-600 \mathrm{~km} \mathrm{~s}^{-1}$.

ESO 428-G14 is an S0/a Seyfert 2 galaxy as classified by de Vaucouleurs et al. (1991). The galaxy is characterized by a twosided radio jet with an [O III] cone also being localized along the jet 
(Falcke et al. 1996). [O III] and $\mathrm{H} \alpha+[\mathrm{N}$ II] narrow-band images obtained with the HST show extended emission well aligned with the radio jet with stronger emission to the north-west as observed in the radio. The ratio $[\mathrm{O} \mathrm{III}] /(\mathrm{H} \alpha+[\mathrm{N}$ II $])$ shows a bipolar structure with larger values to the south-east (Falcke et al. 1996; Falcke, Wilson \& Simpson 1998). Non-circular motions based on the gas kinematics in the inner kiloparsec of the galaxy were reported by Riffel et al. (2006). The galaxy major axis is noted to be aligned with the radio axis. From the radio emission morphology it is suggested that a radio jet is the cause of outflows.

NGC 3081 is an SB0/a galaxy hosting a Seyfert 2 nucleus (Malkan et al. 1998). The galaxy has a weak large-scale bar and a nuclear bar (Buta 1990) followed by four resonance rings - two outer rings, an inner, and a nuclear ring (Buta \& Purcell 1998). Inside the nuclear ring, two spiral arms are observed. A comparison between the continuum adjacent to the [O III] and to the $\mathrm{H} \alpha+[\mathrm{N}$ II $]$ lines showed that the nuclear ring and nuclear spiral arms are sites of recent or ongoing star formation (Ferruit et al. 2000). Radio observations revealed a compact radio source oriented roughly along the northsouth direction (Nagar et al. 1999). Two kinematic components were reported by Schnorr-Müller et al. (2016) based on the optical IFU observations - velocity dispersion of one in the range $\sigma \approx 60$ $100 \mathrm{~km} \mathrm{~s}^{-1}$ over the entire field of view and the other $\sigma \approx 150$ $250 \mathrm{~km} \mathrm{~s}^{-1}$ in the inner $200 \mathrm{pc}$.

NGC 5728 is a Seyfert 2 galaxy classified as $\mathrm{SAB}(\mathrm{r})$ a (de Vaucouleurs et al. 1991). The HST [O III] and $\mathrm{H} \alpha+[\mathrm{N}$ II] narrow- band images by Wilson et al. (1993) show an extended biconical ionisation cone of $\sim 1.8 \mathrm{kpc}$ in extent along the minor axis direction of the host galaxy. According to the kinematic analysis by Rubin (1980), the radial velocities of the central region could be interpreted as an expansion or rotation of the inner galactic disc region or a noncircular motion of a combination of both. There is an asymmetrical bright core at the centre surrounded by star-forming region in a ring of 5 arcsec in radius (Schommer et al. 1988; Mazzuca et al. 2008). The line spectra of the north-west region within 3 arcsec apart from the central nucleus showed double-peaked emission-line profiles (Schommer et al. 1988).

NGC 7213 is an Sa galaxy harbouring a Seyfert 1 AGN (Malkan et al. 1998). The galaxy has a bright nuclear point source with a very faint and featureless accretion disc. The galaxy is almost face on, hence giving a symmetrical appearance. The morphology of the galaxy is characterized by multiple spiral arms with severe dust extinction in the nuclear region. The $8.4 \mathrm{GHz}$ VLA radio map of the galaxy shows an unresolved core (Schmitt et al. 2001) with signs of variability (Blank, Harnett \& Jones 2005). The stellar kinematics from the optical IFU studies by Schnorr-Müller et al. (2014b) of this galaxy shows velocity dispersions of up to $200 \mathrm{~km} \mathrm{~s}^{-1}$ over the entire field of view.

This paper has been typeset from a $\mathrm{T}_{\mathrm{E}} \mathrm{X} / \mathrm{LT} \mathrm{E} \mathrm{X}$ file prepared by the author. 